\title{
RATIONAL SEQUENCES ON DIFFERENT MODELS OF ELLIPTIC CURVES
}

\author{
Gamze Savaş Çelik, Mohammad Sadek and Gökhan Soydan \\ Bursa Uludağ University, Turkey and Sabancı University, Turkey
}

\begin{abstract}
Given a set $S$ of elements in a number field $k$, we discuss the existence of planar algebraic curves over $k$ which possess rational points whose $x$-coordinates are exactly the elements of $S$. If the size $|S|$ of $S$ is either 4,5, or 6, we exhibit infinite families of (twisted) Edwards curves and (general) Huff curves for which the elements of $S$ are realized as the $x$-coordinates of rational points on these curves. This generalizes earlier work on progressions of certain types on some algebraic curves.
\end{abstract}

\section{INTRODUCTION}

An algebraic (affine) plane curve $C$ of degree $d$ over some field $k$ is defined by an equation of the form

$$
\left\{(x, y) \in k^{2}: f(x, y)=0\right\}
$$

where $f$ is a polynomial of degree $d$. The algebraic affine plane curve $C$ can also be extended to the projective plane by homogenising the polynomial $f$. If $P=(x, y)$, then we write $x=x(P)$ and $y=y(P)$.

Studying the set of $k$-rational points on $C, C(k)$, has been subject to extensive research in arithmetic geometry and number theory, especially when $k$ is a number field. For example, if $f$ is a polynomial of degree 2, then one knows that $C$ is of genus 0 , and so if $C$ possesses one rational point then it contains infinitely many such points. If $f$ is of degree 3 , then $C$ is a genus 1 curve if it is smooth. In this case, if $C(k)$ contains one rational point, then it is an elliptic curve, and according to Mordell-Weil Theorem, $C(k)$ is a finitely

2010 Mathematics Subject Classification. 11D25, 11G05, $14 \mathrm{G} 05$.

Key words and phrases. Elliptic curve, Edwards curve, Huff curve, rational sequence, rational point. 
generated abelian group. In particular, $C(k)$ can be written as $T \times \mathbb{Z}^{r}$ where $T$ is the subgroup of points of finite order, and $r \geq 0$ is the rank of $C$ over $k$.

In enumerative geometry, one may pose the following question. Given a set of points $S$ in $k^{2}$, how many algebraic plane curves $C$ of degree $d$ satisfy that $S \subseteq C(k)$ ? It turns out that sometimes the answer is straightforward. For example, given 10 points in $k^{2}$, in order for a cubic curve to pass through these points, a system of 10 linear equations will be obtained by substituting the points of $S$ in

$$
a_{1} x^{3}+a_{2} x^{2} y+a_{3} x^{2}+a_{4} x y^{2}+a_{5} x y+a_{6} x+a_{7} y^{3}+a_{8} y^{2}+a_{9} y+a_{10}=0
$$

and solving for $a_{1}, \cdots, a_{10}$. Therefore, there exists a nontrivial solution to the system if the determinant of the corresponding matrix of coefficients is zero, hence a cubic curve through the points of $S$. Thus, one needs linear algebra to check the existence of algebraic curves of a certain degree through various specified points in $k^{2}$.

In this article, we address the following, relatively harder, question. Given $S \subset k$, are there algebraic curves $C$ of degree $d$ such that for every $x \in S$, $x=x(P)$ for some $P \in C(k)$ ? In other words, $S$ constitutes the $x$-coordinates of a subset of $C(k)$. The latter question can be reformulated to involve $y$ coordinates instead of $x$-coordinates. It is obvious that linear algebra cannot be utilized to attack the problem as substituting with the $x$-values of $S$ will not yield linear equations.

Given a set $S=\left\{x_{1}, x_{2}, \cdots, x_{n}\right\} \subset k$, if $\left(x_{i}, y_{i}\right), i=1, \cdots, n$, are $k$ rational points on an algebraic curve $C$, then these rational points are said to be an $S$-sequence of length $n$. In what follows, we summarize the current state of knowledge for different types of $S$.

We first describe the state-of-art when the elements of $S \subset \mathbb{Q}$ are chosen to form an arithmetic progression, Lee and Vélez ([10]) found infinitely many curves described by $y^{2}=x^{3}+a$ containing $S$-sequences of length 4 . Bremner ([2]) showed that there are infinitely many elliptic curves with $S$-sequences of length 7 and 8. Campbell ([5]) gave a different method to produce infinite families of elliptic curves with $S$-sequences of length 7 and 8 . In addition, he described a method for obtaining infinite families of quartic elliptic curves with $S$-sequences of length 9 , and gave an example of a quartic elliptic curve with an $S$-sequence of length 12 . Ulas ([17]) first described a construction method for an infinite family of quartic elliptic curves on which there exists an $S$-sequence of length 10 . Secondly he showed that there is an infinite family of quartics containing $S$-sequences of length 12. Macleod ([11]) showed that simplifying Ulas' approach may provide a few examples of quartics with $S$ sequences of length 14 . Ulas ([18]) found an infinite family of genus two curves described by $y^{2}=f(x)$ where $\operatorname{deg}(f(x))=5$ possessing $S$-sequences of length 11. Alvarado ([1]) showed the existence of an infinite family of such curves with $S$-sequences of length 12 . Moody ([12]) found an infinite number of 
Edwards curves with an $S$-sequence of length 9 . He also asked whether any such curve will allow an extension to an $S$-sequence of length 11 . Bremner ([3]) showed that such curves do not exist. Also, Moody ([14]) found an infinite number of Huff curves with $S$-sequences of length 9, and Choudhry ([6]) extended Moody's result to find several Huff curves with $S$-sequences of length 11.

Now we consider the case when the elements of $S$ form a geometric progression, Bremner and Ulas ([4]) obtained an infinite family of elliptic curves with $S$-sequences of length 4 , and they also pointed out infinitely many elliptic curves with $S$-sequences of length 5 . Ciss and Moody ([13]) found infinite families of twisted Edwards curves with $S$-sequences of length 5 and Edwards curves with $S$-sequences of length 4 . When the elements of $S \subset \mathbb{Q}$ are consecutive squares, Kamel and Sadek ([9]) constructed infinitely many elliptic curves given by the equation $y^{2}=a x^{3}+b x+c$ with $S$-sequences of length 5. When the elements of $S \subset \mathbb{Q}$ are consecutive cubes, Çelik and Soydan ([7]) found infinitely many elliptic curves of the form $y^{2}=a x^{3}+b x+c$ with $S$-sequences of length 5 .

In the present work, we consider the following families of elliptic curves due to the symmetry enjoyed by the equations defining them: (twisted) Edwards curves and (general) Huff curves. Given an arbitrary subset $S$ of a number field $k$, we tackle the general question of the existence of infinitely many such curves with an $S$-sequence when there is no restriction on the elements of $S$. We provide explicit examples when the length of the $S$-sequence is 4,5 , or 6 . This is achieved by studying the existence of rational points on certain quadratic and elliptic surfaces.

\section{EdWARds CURVES WITH $S$-SEQUENCES OF LENGTH 6}

Throughout this work, $k$ will be a number field unless otherwise stated. An Edwards curve over $k$ is defined by

$$
E_{d}: x^{2}+y^{2}=1+d x^{2} y^{2},
$$

where $d$ is a non-zero element in $k$. It is clear that the points $(x, y)=$ $(-1,0),(0, \pm 1),(1,0) \in E_{d}(k)$. We show that given any set

$$
S=\left\{s_{-1}=-1, s_{0}=0, s_{1}=1, s_{2}, s_{3}, s_{4}\right\} \subset k,
$$

$s_{i} \neq s_{j}$ if $i \neq j$, there are infinitely many Edwards curves $E_{d}$ that possess rational points whose $x$-coordinates are $s_{i},-1 \leq i \leq 4$, i.e., the set $S$ is realized as $x$-coordinates in $E_{d}(k)$. In other words, there are infinitely many Edwards curves that possess an $S$-sequence.

We start with assuming that $s_{2}$ is the $x$-coordinate of a point in $E_{d}(k)$, then one must have $y^{2}=\frac{s_{2}^{2}-1}{s_{2}^{2} d-1}$, or $s_{2}^{2} d-1=\left(s_{2}^{2}-1\right) p^{2}$ for some $p=1 / y \in k$. 
Similarly, if $s_{3}$ is the $x$-coordinate of a point in $E_{d}(k)$, then $y^{2}=\frac{s_{3}^{2}-1}{s_{3}^{2} d-1}$, or $s_{3}^{2} d-1=\left(s_{3}^{2}-1\right) q^{2}$. So

$$
d=\frac{\left(s_{2}^{2}-1\right) p^{2}+1}{s_{2}^{2}}=\frac{\left(s_{3}^{2}-1\right) q^{2}+1}{s_{3}^{2}} .
$$

Thus we have the following quadratic curve

$$
s_{3}^{2}\left[\left(s_{2}^{2}-1\right) p^{2}+1\right]-s_{2}^{2}\left[\left(s_{3}^{2}-1\right) q^{2}+1\right]=0
$$

on which we have the rational point $(p, q)=(1,1)$. Parametrizing the rational points on the latter quadratic curve yields

$$
\begin{aligned}
& p=\frac{2 t s_{2}^{2}-t^{2} s_{2}^{2}-s_{3}^{2}+s_{2}^{2} s_{3}^{2}-2 t s_{2}^{2} s_{3}^{2}+t^{2} s_{2}^{2} s_{3}^{2}}{-t^{2} s_{2}^{2}+s_{3}^{2}-s_{2}^{2} s_{3}^{2}+t^{2} s_{2}^{2} s_{3}^{2}} \\
& q=-\frac{\left(-1+s_{2}^{2}\right) s_{3}^{2}-2 t\left(-1+s_{2}^{2}\right) s_{3}^{2}+t^{2} s_{2}^{2}\left(-1+s_{3}^{2}\right)}{-\left(-1+s_{2}^{2}\right) s_{3}^{2}+t^{2} s_{2}^{2}\left(-1+s_{3}^{2}\right)} .
\end{aligned}
$$

Therefore, fixing $s_{2}$ and $s_{3}$ in $k$, one sees that $p$ and $q$ lie in $k(t)$. Now we obtain the following result.

THEOREM 2.1. Let $s_{-1}=-1, s_{0}=0, s_{1}=1, s_{2}, s_{3}$ and $s_{4}, s_{i} \neq s_{j}$ if $i \neq j$, be a sequence in $\mathbb{Z}$ such that

$$
h\left(s_{2}, s_{3}\right)=-3+4 s_{3}^{2}+s_{2}^{4} s_{3}^{4}+s_{2}^{2}\left(4-6 s_{3}^{2}\right) \neq 0
$$

where either $g_{1}\left(s_{2}, s_{3}\right) / h\left(s_{2}, s_{3}\right)^{2}$ or $g_{2}\left(s_{2}, s_{3}\right) / h\left(s_{2}, s_{3}\right)^{3}$ are not integers, $g_{1}$ and $g_{2}$ are defined in (2.3). There are infinitely many Edwards curves described by

$$
E_{d}: x^{2}+y^{2}=1+d x^{2} y^{2}, \quad d \in \mathbb{Q}
$$

on which $s_{i},-1 \leq i \leq 4$, are the $x$-coordinates of rational points in $E_{d}(\mathbb{Q})$. In other words, there are infinitely many Edwards curves that possess an $S$ sequence where $S=\left\{s_{i}:-1 \leq i \leq 4\right\}$.

PROOF. Substituting the value for $p$ in $d=\frac{\left(s_{2}^{2}-1\right) p^{2}+1}{s_{2}^{2}}$ yields that

$$
\begin{aligned}
(- & \left.t^{2} s_{2}^{2}+s_{3}^{2}-s_{2}^{2} s_{3}^{2}+t^{2} s_{2}^{2} s_{3}^{2}\right)^{2} d \\
= & \left(s_{3}^{4}-2 s_{2}^{2} s_{3}^{4}+s_{2}^{4} s_{3}^{4}\right)+\left(4 s_{3}^{2}-8 s_{2}^{2} s_{3}^{2}+4 s_{2}^{4} s_{3}^{2}-4 s_{3}^{4}+8 s_{2}^{2} s_{3}^{4}-4 s_{2}^{4} s_{3}^{4}\right) t \\
& \quad+\left(-4 s_{2}^{2}+4 s_{2}^{4}-4 s_{3}^{2}+14 s_{2}^{2} s_{3}^{2}-10 s_{2}^{4} s_{3}^{2}+4 s_{3}^{4}-10 s_{2}^{2} s_{3}^{4}+6 s_{2}^{4} s_{3}^{4}\right) t^{2} \\
& \quad+\left(4 s_{2}^{2}-4 s_{2}^{4}-8 s_{2}^{2} s_{3}^{2}+8 s_{2}^{4} s_{3}^{2}+4 s_{2}^{2} s_{3}^{4}-4 s_{2}^{4} s_{3}^{4}\right) t^{3}+\left(s_{2}^{4}-2 s_{2}^{4} s_{3}^{2}+s_{2}^{4} s_{3}^{4}\right) t^{4} .
\end{aligned}
$$

Thus, for fixed values of $s_{2}$ and $s_{3}$, we have $d \in \mathbb{Q}(t)$.

Now we show the existence of infinitely many values of $t$ such that $s_{4}$ is the $x$-coordinate of a rational point on $E_{d}$. In fact, we will show that $t$ can be chosen to be the $x$-coordinate of a rational point on an elliptic curve with positive Mordell-Weil rank, hence the existence of infinitely many such 
possible values for $t$. Forcing $\left(s_{4}, r\right)$ to be a point in $E_{d}(\mathbb{Q})$ for some rational $r$ yields that

$$
r^{2}=\frac{s_{4}^{2}-1}{s_{4}^{2} d-1}=\left(A_{0}+A_{1} t+A_{2} t^{2}+A_{3} t^{3}+A_{4} t^{4}\right) / B(t)^{2},
$$

where $A_{i} \in \mathbb{Z}$ and $B(t)=-t^{2} s_{2}^{2}+t^{2} s_{2}^{2} s_{3}^{2}+s_{3}^{2}-s_{2}^{2} s_{3}^{2}$. This implies that $A_{0}+A_{1} t+A_{2} t^{2}+A_{3} t^{3}+A_{4} t^{4}$ must be a rational square. This yields the elliptic curve $C$ defined by

$$
z^{2}=A_{0}+A_{1} t+A_{2} t^{2}+A_{3} t^{3}+A_{4} t^{4},
$$

with the following rational point

$$
(t, z)=\left(0, s_{3}^{2}\left(s_{2}^{2}-1\right)\right) .
$$

The latter elliptic curve is isomorphic to the elliptic curve described by the Weierstrass equation $E_{I, J}: y^{2}=x^{3}-27 I x-27 J$ where

$$
\begin{aligned}
& I=12 A_{0} A_{4}-3 A_{1} A_{3}+A_{2}^{2} \\
& J=72 A_{0} A_{2} A_{4}+9 A_{1} A_{2} A_{3}-27 A_{1}^{2} A_{4}-27 A_{0} A_{3}^{2}-2 A_{2}^{3},
\end{aligned}
$$

see for example $[16, \S 2]$. The latter elliptic curve has the following rational point

$$
P=\left(-12\left(-1+s_{2}^{2}\right)\left(-1+s_{3}^{2}\right)\left(-3+s_{2}^{2}+s_{3}^{2}\right),-216\left(-1+s_{2}^{2}\right)^{2}\left(-1+s_{3}^{2}\right)^{2}\right) .
$$

One notices that the coordinates of $3 P$ are rational functions. Indeed,

$$
3 P=\left(\frac{g_{1}\left(s_{2}, s_{3}\right)}{h\left(s_{2}, s_{3}\right)^{2}}, \frac{g_{2}\left(s_{2}, s_{3}\right)}{h\left(s_{2}, s_{3}\right)^{3}}\right), \quad \text { where } g_{1}, g_{2} \in \mathbb{Q}\left[s_{2}, s_{3}\right]
$$

and

$$
h\left(s_{2}, s_{3}\right)=-3+4 s_{3}^{2}+s_{2}^{4} s_{3}^{4}+s_{2}^{2}\left(4-6 s_{3}^{2}\right) .
$$

Hence, as long as $h\left(s_{2}, s_{3}\right) \neq 0$, and $g_{1} / h^{2} \notin \mathbb{Z}$ or $g_{2} / h^{3} \notin \mathbb{Z}$, one sees that $3 P$ is a point of infinite order by virtue of Lutz-Nagell Theorem. Thus, $P$ itself is a point of infinite order. It follows that $E_{I, J}$ is of positive Mordell-Weil rank. Since $C$ is isomorphic to $E_{I, J}$, it follows that $C$ is also of positive MordellWeil rank. Therefore, there are infinitely many rational points $(t, z) \in C(\mathbb{Q})$, each giving rise to a value for $d$, by substituting in (2.2), hence an Edwards curve $E_{d}$ possessing the aforementioned rational points. That infinitely many of these curves are pairwise non-isomorphic over $\mathbb{Q}$ follows, for instance, from [8, Proposition 6.1].

\section{Twisted Edwards curves with $S$-Sequences of Length 4}

A Twisted Edwards curve over $k$ is given by

$$
E_{a, d}: a x^{2}+y^{2}=1+d x^{2} y^{2},
$$

where $a$ and $d$ are nonzero elements in $k$. Note that the point $(x, y)=(0, \pm 1) \in$ $E_{a, d}(k)$. Given a set $\left\{u_{0}=0, u_{1}, u_{2}, u_{3}\right\} \subset k, u_{i} \neq u_{j}$ if $i \neq j$, we prove that 
there are infinitely many twisted Edwards curves $E_{a, d}$ for which $S$ is realized as the $x$-coordinates of rational points on $E_{a, d}$.

We begin by assuming that $u_{1}$ is the $x$-coordinate of a point in $E_{a, d}(k)$, then one must get $y^{2}=\frac{a u_{1}^{2}-1}{u_{1}^{2} d-1}$, or $u_{1}^{2} d-1=\left(a u_{1}^{2}-1\right) i^{2}$ for some $i \in k$.

Now, if $u_{2}$ is the $x$-coordinate of a point in $E_{a, d}(k)$, then $y^{2}=\frac{a u_{2}^{2}-1}{u_{2}^{2} d-1}$ or $u_{2}^{2} d-1=\left(a u_{2}^{2}-1\right) j^{2}$. So

$$
d=\frac{\left(a u_{1}^{2}-1\right) i^{2}+1}{u_{1}^{2}}=\frac{\left(a u_{2}^{2}-1\right) j^{2}+1}{u_{2}^{2}} .
$$

Hence we obtain the following quadratic surface

$$
u_{2}^{2}\left[\left(a u_{1}^{2}-1\right) i^{2}+1\right]-u_{1}^{2}\left[\left(a u_{2}^{2}-1\right) j^{2}+1\right]=0,
$$

on which we have the rational point $(i, j)=(1,1)$. Solving the above quadratic surface gives the following

$$
\begin{aligned}
& i=\frac{-a u_{1}^{2} u_{2}^{2}+u_{2}^{2}+2 t a u_{1}^{2} u_{2}^{2}-2 t u_{1}^{2}-a t^{2} u_{1}^{2} u_{2}^{2}+u_{1}^{2} t^{2}}{a u_{1}^{2} u_{2}^{2}-u_{2}^{2}-a t^{2} u_{1}^{2} u_{2}^{2}+u_{1}^{2} t^{2}}, \\
& j=\frac{-2 a t u_{1}^{2} u_{2}^{2}+2 t u_{2}^{2}+a t^{2} u_{1}^{2} u_{2}^{2}-u_{1}^{2} t^{2}+a u_{1}^{2} u_{2}^{2}-u_{2}^{2}}{a u_{1}^{2} u_{2}^{2}-u_{2}^{2}-a t^{2} u_{1}^{2} u_{2}^{2}+u_{1}^{2} t^{2}} .
\end{aligned}
$$

Now we get the following result.

TheOrem 3.1. Let $u_{0}=0, u_{1}, u_{2}$ and $u_{3}, u_{i} \neq u_{j}$ if $i \neq j$, be a sequence in $\mathbb{Z}$ such that $h\left(u_{1}, u_{2}\right) \neq 0$, and either $g_{1}\left(s_{2}, s_{3}\right) / h\left(s_{2}, s_{3}\right)^{2}$ or $g_{2}\left(s_{2}, s_{3}\right) / h\left(s_{2}, s_{3}\right)^{3}$ are not integers, where $h, g_{1}, g_{2}$ are defined in (3.3). There are infinitely many twisted Edwards curves described by

$$
E_{a, d}: a x^{2}+y^{2}=1+d x^{2} y^{2}, \quad d \in \mathbb{Q}, a \in \mathbb{Q}^{\times} \text {is arbitrary }
$$

on which $u_{i}, 0 \leq i \leq 3$, are the $x$-coordinates of rational points in $E(\mathbb{Q})$. In other words, there are infinitely many twisted Edwards curves that possess an $S$-sequence where $S=\left\{u_{i}: 0 \leq i \leq 3\right\}$.

Proof. Substituting the expression for $i$ in $d=\frac{\left(a u_{1}^{2}-1\right) i^{2}+1}{u_{1}^{2}}$ gives that

$$
\begin{aligned}
\left(a u_{1}^{2}\right. & \left.u_{2}^{2}-u_{2}^{2}-a t^{2} u_{1}^{2} u_{2}^{2}+u_{1}^{2} t^{2}\right)^{2} d \\
= & \left(u_{1}^{4} a^{3} u_{2}^{4}-2 u_{1}^{4} a^{2} u_{2}^{2}+u_{1}^{4} a\right) t^{4}+\left(-8 a u_{1}^{2} u_{2}^{2}+4 u_{1}^{2}+4 u_{1}^{2} a^{2} u_{2}^{4}-4 u_{1}^{4} a\right. \\
& \left.-4 u_{1}^{4} a^{3} u_{2}^{4}+8 u_{1}^{4} a^{2} u_{2}^{2}\right) t^{3}+\left(-4 u_{1}^{2}-10 u_{1}^{2} a^{2} u_{2}^{4}+14 a u_{1}^{2} u_{2}^{2}+6 u_{1}^{4} a^{3} u_{2}^{4}\right. \\
& \left.-4 u_{2}^{2}-10 u_{1}^{4} a^{2} u_{2}^{2}+4 u_{1}^{4} a+4 a u_{2}^{4}\right) t^{2}+\left(4 u_{2}^{2}+8 u_{1}^{2} a^{2} u_{2}^{4}-8 a u_{1}^{2} u_{2}^{2}\right. \\
& \left.+4 u_{1}^{4} a^{2} u_{2}^{2}-4 a u_{2}^{4}-4 u_{1}^{4} a^{3} u_{2}^{4}\right) t+u_{1}^{4} a^{3} u_{2}^{4}-2 u_{1}^{2} a^{2} u_{2}^{4}+a u_{2}^{4} .
\end{aligned}
$$


Then, assuming $\left(u_{3}, \ell\right) \in E(\mathbb{Q})$ yields

$$
\ell^{2}=\frac{a u_{3}^{2}-1}{d u_{3}^{2}-1}=\left(C_{0}+C_{1} t+C_{2} t^{2}+C_{3} t^{3}+C_{4} t^{4}\right) / D(t)^{2},
$$

where $C_{i} \in \mathbb{Q}$ and $D(t)=a u_{1}^{2} u_{2}^{2}-u_{2}^{2}-a t^{2} u_{1}^{2} u_{2}^{2}+u_{1}^{2} t^{2}$.

For the latter equation to be satisfied, one needs to find rational points on the elliptic curve $C^{\prime}$ defined by

$$
z^{2}=C_{0}+C_{1} t+C_{2} t^{2}+C_{3} t^{3}+C_{4} t^{4}
$$

that possesses the rational point

$$
(t, z)=\left(0, u_{2}^{2}\left(a u_{1}^{2}-1\right)\right) .
$$

The latter elliptic curve is isomorphic to the elliptic curve described by the Weierstrass equation $E_{I, J}: y^{2}=x^{3}-27 I x-27 J$ where

$$
\begin{aligned}
& I=12 C_{0} C_{4}-3 C_{1} C_{3}+C_{2}^{2}, \\
& J=72 C_{0} C_{2} C_{4}+9 C_{1} C_{2} C_{3}-27 C_{1}^{2} C_{4}-27 C_{0} C_{3}^{2}-2 C_{2}^{3},
\end{aligned}
$$

see for example $[16, \S 2]$. The latter elliptic curve has the following rational point

$Q=$

$$
\left(-12\left(-1+a u_{2}^{2}\right)\left(-1+a u_{1}^{2}\right)\left(-3+a u_{2}^{2}+u_{1}^{2}\right),-216\left(-1+a u_{2}^{2}\right)^{2}\left(-1+a u_{1}^{2}\right)^{2}\right) .
$$

One notices that the coordinates of $3 Q$ are rational functions. In fact,

$$
3 Q=\left(\frac{g_{1}\left(u_{1}, u_{2}\right)}{h\left(u_{1}, u_{2}\right)^{2}}, \frac{g_{2}\left(u_{1}, u_{2}\right)}{h\left(u_{1}, u_{2}\right)^{3}}\right), \quad \text { where } g_{1}, g_{2} \in \mathbb{Q}\left[u_{1}, u_{2}\right]
$$

and

$$
\begin{aligned}
h\left(u_{1}, u_{2}\right)= & -27-72 u_{1}^{2}+36 u_{1}^{4}+18 u_{1}^{2} u_{2}^{2}-12 u_{1}^{4} u_{2}^{2}-18 u_{2}^{4}+12 u_{1}^{2} u_{2}^{4} \\
& +u_{1}^{4} u_{2}^{4}-2 u_{1}^{2} u_{2}^{6}+u_{2}^{8}+a\left(36 u_{1}^{2}-12 u_{1}^{4}-24 u_{1}^{2}\left(-3+u_{1}^{2}\right)\right. \\
& +36 u_{2}^{2}+72 u_{1}^{2} u_{2}^{2}-24 u_{1}^{4} u_{2}^{2}-12 u_{1}^{2} u_{2}^{4}+4 u_{1}^{4} u_{2}^{4} \\
& \left.-4\left(-3+u_{1}^{2}\right) u_{2}^{6}\right)+a^{2}\left(-144 u_{1}^{2} u_{2}^{2}+36 u_{1}^{4} u_{2}^{2}+18 u_{2}^{4}\right. \\
& \left.-36 u_{1}^{2} u_{2}^{4}+4 u_{1}^{4} u_{2}^{4}+2 u_{1}^{2} u_{2}^{6}-2 u_{2}^{8}\right)+a^{3}\left(36 u_{1}^{2} u_{2}^{4}\right. \\
& \left.+4\left(-3+u_{1}^{2}\right) u_{2}^{6}\right)+a^{4} u_{2}^{8} .
\end{aligned}
$$

Therefore, as long as $h\left(u_{1}, u_{2}\right) \neq 0$ and $g_{1} / h^{2} \notin \mathbb{Z}$ or $g_{2} / h^{3} \notin \mathbb{Z}$, one sees that $E_{I, J}$ is of positive Mordell-Weil rank where the point $Q$ is of infinite order. Since $C^{\prime}$ is isomorphic to $E_{I, J}$, it follows that $C^{\prime}$ is also of positive Mordell-Weil rank. Hence, there are infinitely many rational points $(t, z) \in$ $C^{\prime}(\mathbb{Q})$, each giving rise to a value for $d$, by substituting in $(3.2)$, therefore a twisted Edwards curve $E_{a, d}$ possessing the aforementioned rational points. That infinitely many of these curves are pairwise non-isomorphic over $\mathbb{Q}$ again follows from [8, Proposition 6.1]. 
REMARK 3.2. Since $(0,-1),(0,1)$ are rational points on any twisted Edwards curve, one can show that if $u_{-1}=-1, u_{1}=1, u_{2}, u_{3}$ and $u_{4}, u_{i} \neq u_{j}$ if $i \neq j$, is a sequence in $\mathbb{Z}$, there are infinitely many Edwards curves on which $u_{i}, i \in\{-1,1,2,3,4\}$, are the $y$-coordinates of rational points in $E_{a, d}(\mathbb{Q})$.

\section{Huff Curves With $S$-SEquences of Length 5}

A Huff curve over a number field $k$ is defined by

$$
H_{a, b}: a x\left(y^{2}-1\right)=b y\left(x^{2}-1\right),
$$

with $a^{2} \neq b^{2}$. Note that the points $(x, y)=(-1, \pm 1),(0,0),(1, \pm 1)$ are in $H_{a, b}(k)$. We prove that given $s_{-1}=-1, s_{0}=0, s_{1}=1, s_{2}, s_{3} \in k, s_{i} \neq s_{j}$ if $i \neq j$, there are infinitely many Huff curves on which these numbers are realized as the $x$-coordinates of rational points.

Assuming $\left(s_{2}, p\right)$ and $\left(s_{3}, q\right)$ are two points on $H_{a, b}$ yields

$$
a s_{2}\left(p^{2}-1\right)=b p\left(s_{2}^{2}-1\right),
$$

and

$$
a s_{3}\left(q^{2}-1\right)=b q\left(s_{3}^{2}-1\right),
$$

respectively. Using (4.2) and (4.3), one obtains

$$
\frac{s_{2}\left(p^{2}-1\right)}{s_{3}\left(q^{2}-1\right)}=\frac{p\left(s_{2}^{2}-1\right)}{q\left(s_{3}^{2}-1\right)},
$$

therefore, one needs to consider the curve

$$
C^{\prime}: A p q^{2}-A p-B q p^{2}+B q=0,
$$

where $A=s_{3} s_{2}^{2}-s_{2}$ and $B=s_{2} s_{3}^{2}-s_{2}$. Dividing both sides of the above equality by $q^{3}$ gives

$$
A \frac{p}{q}-A \frac{p}{q} \frac{1}{q^{2}}-B\left(\frac{p}{q}\right)^{2}+B \frac{1}{q^{2}}=0
$$

Substituting $x=\frac{p}{q}$ and $y=\frac{1}{q^{2}}$ in the above equation yields the following quadratic curve

$$
A x-A x y-B x^{2}+B y=0,
$$

on which we have the rational point $(x, y)=(1,1)$. Parametrizing the rational points on the latter quadratic curve gives

$$
\begin{aligned}
& x=\frac{B t-B}{A t+B}, \\
& y=\frac{A t(1-t)+B(1-t)^{2}}{A t+B} .
\end{aligned}
$$

Now we have the following result. 
Theorem 4.1. Let $s_{-1}=-1, s_{0}=0, s_{1}=1, s_{2}, s_{3}, s_{m} \neq s_{n}$ if $m \neq n$, be a sequence in $\mathbb{Z}$ such that

$$
h=-4+A^{2}-3 A B+B^{2} \neq 0
$$

where $A$ and $B$ are defined as above, and either $g_{1} / h^{2}$ or $g_{2} / h^{3}$ are not integers, where $g_{1}, g_{2}$ are defined in (4.6). There are infinitely many Huff curves described by

$$
H_{a, b}: a x\left(y^{2}-1\right)=b y\left(x^{2}-1\right), \quad a, b \in \mathbb{Q}, \quad a^{2} \neq b^{2}
$$

on which $s_{m},-1 \leq m \leq 3$, are the $x$-coordinates of rational points in $H_{a, b}(\mathbb{Q})$. In other words, there are infinitely many Huff curves that possess an $S$-sequence where $S=\left\{s_{i}:-1 \leq i \leq 3\right\}$.

Proof. Using the equalities (4.4) and (4.5), we obtain the following

$$
\begin{aligned}
& p^{2}=\frac{x^{2}}{y}=\frac{B^{2}(-1+t)}{(B(-1+t)-A t)(B+A t)}, \\
& q^{2}=\frac{1}{y}=\frac{(B+A t)}{(-1+t)(B(-1+t)-A t)} .
\end{aligned}
$$

In both cases we need $(B+A t)(-1+t)(B(-1+t)-A t)$ to be a square or in other words we need $t$ to be the $x$-coordinate of a rational point on the elliptic curve $C^{\prime \prime}$ defined by

$$
z^{2}=(A t+B)(t-1)(t(B-A)-B),
$$

with the following $k$-rational point $(t, z)=(0, B)$. The latter curve can be described by the following equation

$$
Y^{2}=X^{3}+\left((B-A)^{2}-A B\right) X^{2}-2 A B(B-A)^{2} X+A^{2} B^{2}(B-A)^{2},
$$

where $A(B-A) t=X$ and $A(B-A) z=Y$. This curve has the rational point

$$
R=(X, Y)=(0, A B(B-A)) .
$$

Observing that

$$
3 R=\left(\frac{g_{1}(A, B)}{h(A, B)^{2}}, \frac{g_{2}(A, B)}{h(A, B)^{3}}\right)
$$

where $h(A, B)=-4+A^{2}-3 A B+B^{2}$, one concludes as in the proof of Theorem 2.1.

\section{General Huff curves with $S$-Sequences of length 4}

A general Huff curve over a number field $k$ is defined by

$$
G_{a, b}: x\left(a y^{2}-1\right)=y\left(b x^{2}-1\right)
$$

where $a, b \in k$ and $a b(a-b) \neq 0$. It is clear that the point $(x, y)=(0,0) \in$ $G_{a, b}(k)$. We show that given $u_{0}=0, u_{1}, u_{2}, u_{3}$ in $k, u_{i} \neq u_{j}$ if $i \neq j$, there 
are infinitely many general Huff curves over which these points are realized as the $x$-coordinates of rational points.

We start by assuming that if $u_{1}$ is the $x$-coordinates of a point in $G_{a, b}(k)$, then one must have $\frac{a y^{2}-1}{y}=\frac{b u_{1}^{2}-1}{u_{1}}$ or $\frac{a-i^{2}}{i}=\frac{b u_{1}^{2}-1}{u_{1}}$ for some $i \in k$.

Similarly, if $u_{2}$ is the $x$-coordinate of a point in $G_{a, b}$, then $\frac{a y^{2}-1}{y}=$ $\frac{b u_{2}^{2}-1}{u_{2}}$ or $\frac{a-j^{2}}{j}=\frac{b u_{2}^{2}-1}{u_{2}}$ for some $j \in k$. Thus, one obtains

$$
a=\frac{\left(b u_{1}^{2}-1\right) i+u_{1} i^{2}}{u_{1}}=\frac{\left(b u_{2}^{2}-1\right) j+u_{2} j^{2}}{u_{2}},
$$

which gives the following quadratic curve

$$
S: A i^{2}+B j^{2}+C i z+D j z=0,
$$

where $A=-u_{1} u_{2}, B=u_{1} u_{2}, C=-u_{1}^{2} u_{2} b+u_{2}, D=b u_{1} u_{2}^{2}-u_{1}$. Then consider the line

$$
m P+n Q=(n p: n q: m+n r)
$$

connecting the rational points $P=(i: j: z)=(0: 0: 1)$ and $Q=(p: q: r)$ lying on $S \subset \mathbb{P}^{2}$. The intersection of $S$ and $m P+n Q$ yields the quadratic equation

$$
n^{2}\left(A p^{2}+B q^{2}+C p r+D q r\right)+m n(C p+D q)=0 .
$$

Using $P$ and $Q$ lying on $S$, one solves this quadratic equation and obtains formulae for the solution $(i: j: z)$ with the following parametrization:

$$
\begin{aligned}
& i=n p=C p^{2}+D p q, \\
& j=n q=C p q+D q^{2}, \\
& z=m+n r=-A p^{2}-B q^{2} .
\end{aligned}
$$

Now we obtain the following result.

TheOREM 5.1. Let $u_{0}=0, u_{1}, u_{2}$ and $u_{3}, u_{i} \neq u_{j}$ if $i \neq j$, be a sequence in $k$. There are infinitely many general Huff curves described by

$$
G_{a, b}: x\left(a y^{2}-1\right)=y\left(b x^{2}-1\right), \quad a, b \in k, \quad a b(a-b) \neq 0 .
$$

on which $u_{i}, 0 \leq i \leq 3$, are the $x$-coordinates of rational points in $G_{a, b}(k)$. In other words, there are infinitely many general Huff curves that possess an $S$-sequence where $S=\left\{u_{i}: 0 \leq i \leq 3\right\}$.

Proof. Substituting the value for $i$ in $a=\frac{\left(b u_{1}^{2}-1\right) i+u_{1} i^{2}}{u_{1}}$ yields that

$$
\begin{aligned}
a= & u_{2}^{2}\left(b u_{1}{ }^{2}-1\right)^{2} p^{4}-2 u_{1} u_{2}\left(b u_{2}{ }^{2}-1\right)\left(b u_{1}^{2}-1\right) p^{3} q \\
& +u_{1}^{2}\left(b u_{2}^{2}-1\right)^{2} p^{2} q^{2}-\frac{u_{2}\left(b u_{1}^{2}-1\right)^{2}}{u_{1}} p^{2}+\left(b u_{2}{ }^{2}-1\right)\left(b u_{1}{ }^{2}-1\right) p q .
\end{aligned}
$$


Now we assume that $\left(u_{3}, \ell\right) \in G_{a, b}(k)$. This yields that

$$
\begin{aligned}
& p u_{3}\left(b p^{2} u_{1}{ }^{3} u_{2}-b p q u_{1}{ }^{2} u_{2}{ }^{2}-p^{2} u_{1} u_{2}+p q u_{1}^{2}-b u_{1}^{2}+1\right) \\
& \left(b p u_{1}^{2} u_{2}-b q u_{1} u_{2}^{2}-p u_{2}+q u_{1}\right) \ell^{2}-u_{1}\left(b u_{3}^{2}-1\right) \ell-u_{1} u_{3}=0 .
\end{aligned}
$$

This can be rewritten as

$$
\begin{aligned}
& Z^{2}\left(b^{2} p^{4} u_{1}^{5} u_{2}^{2} u_{3}-2 b p^{4} u_{1}^{3} u_{2}^{2} u_{3}-b^{2} p^{2} u_{1}^{4} u_{2} u_{3}+p^{4} u_{1} u_{2}^{2} u_{3}+2 b p^{2} u_{1}^{2} u_{2} u_{3}\right. \\
& \left.\quad-p^{2} u_{2} u_{3}\right)+q Z\left(-2 b^{2} p^{3} u_{1}^{4} u_{2}^{3} u_{3}+2 b p^{3} u_{1}^{4} u_{2} u_{3}+2 b p^{3} u_{1}^{2} u_{2}^{3} u_{3}+b^{2} p u_{1}^{3} u_{2}^{2} u_{3}\right. \\
& \left.\quad-2 p^{3} u_{1}^{2} u_{2} u_{3}-b p u_{1}^{3} u_{3}-b p u_{1} u_{2}^{2} u_{3}+p u_{1} u_{3}\right)+q^{2} p^{2} u_{1}^{3} u_{3}\left(b u_{2}^{2}-1\right)^{2} \\
& \quad-T Z u_{1}\left(b u_{3}^{2}-1\right)-T^{2} u_{1} u_{3}=0,
\end{aligned}
$$

where $T=1 / \ell$. One sees that the rational point $P=(q: T: Z)=(1: 0:$ $\left.u_{1}\left(-1+b u_{2}^{2}\right) / p u_{2}\left(-1+b u_{1}^{2}\right)\right)$ lies on the quadratic curve above, hence we may parametrize the rational points on the quadratic curve above. This is obtained by considering the intersection of the line $d P+e Q$ where $Q=\left(q_{1}: q_{2}: q_{3}\right)$ is a point on the quadratic curve. In fact, this yields that

$$
\begin{aligned}
d= & p u_{2}\left(b u_{1}^{2}-1\right)\left(q_{3}{ }^{2} b^{2} p^{4} u_{1}{ }^{5} u_{2}{ }^{2} u_{3}-2 q_{3}{ }^{2} b p^{4} u_{1}{ }^{3} u_{2}{ }^{2} u_{3}\right. \\
& -q_{3}{ }^{2} b^{2} p^{2} u_{1}{ }^{4} u_{2} u_{3}+q_{3}{ }^{2} p^{4} u_{1} u_{2}{ }^{2} u_{3}+2 q_{3}{ }^{2} b p^{2} u_{1}{ }^{2} u_{2} u_{3} \\
& -q_{3}{ }^{2} p^{2} u_{2} u_{3}-u_{1} q_{2} q_{3} b u_{3}{ }^{2}+u_{1} q_{2} q_{3}+p^{2} u_{1}{ }^{3} u_{3} q_{1}{ }^{2} b^{2} u_{2}{ }^{4} \\
& -2 p^{2} u_{1}{ }^{3} u_{3} q_{1}{ }^{2} b u_{2}{ }^{2}+p^{2} u_{1}{ }^{3} u_{3} q_{1}^{2}-2 q_{1} q_{3} b^{2} p^{3} u_{1}{ }^{4} u_{2}{ }^{3} u_{3} \\
& +2 q_{1} q_{3} b p^{3} u_{1}{ }^{4} u_{2} u_{3}+2 q_{1} q_{3} b p^{3} u_{1}{ }^{2} u_{2}{ }^{3} u_{3}+q_{1} q_{3} b^{2} p u_{1}{ }^{3} u_{2}{ }^{2} u_{3} \\
& -2 q_{1} q_{3} p^{3} u_{1}{ }^{2} u_{2} u_{3}-q_{1} q_{3} b p u_{1}{ }^{3} u_{3}-q_{1} q_{3} b p u_{1} u_{2}{ }^{2} u_{3}+q_{1} q_{3} p u_{1} u_{3} \\
& \left.-u_{1} u_{3} q_{2}{ }^{2}\right), \\
e & u_{1}\left(b u_{2}{ }^{2}-1\right)\left(-p u_{1}{ }^{3} u_{3} q_{1} b^{2} u_{2}{ }^{2}+p^{2} u_{3} q_{3} u_{2} b^{2} u_{1}{ }^{4}+p u_{1} u_{3} q_{1} b u_{2}{ }^{2}\right. \\
& \left.-2 p^{2} u_{3} q_{3} u_{2} b u_{1}{ }^{2}+p u_{1}{ }^{3} u_{3} q_{1} b+u_{1} q_{2} b u_{3}{ }^{2}+p^{2} u_{3} q_{3} u_{2}-u_{1} q_{2}-p u_{1} u_{3} q_{1}\right) .
\end{aligned}
$$

ACKNOWLEDGEMENTS.

We would like to thank the referees for carefully reading our manuscript and for giving such constructive comments which substantially helped improving the presentation of the paper.

\section{REFERENCES}

[1] A. Alvarado, An arithmetic progression on quintic curves, J. Integer Seq. 12 (2009), Article 09.7.3., 6 pp.

[2] A. Bremner, On arithmetic progressions on elliptic curves, Experiment Math. 8, (1999), 409-413.

[3] A. Bremner, Arithmetic progressions on Edwards curves, J. Integer Seq. 16 (2013), Article 13.8.5., 5 pp. 
[4] A. Bremner and M. Ulas, Rational points in geometric progressions on certain hyperelliptic curves, Publ. Math. Debrecen 82 (2013), 669-683.

[5] G. Campbell, A note on arithmetic progressions on elliptic curves, J. Integer Seq. 6 (2003), Article 03.1.3., 5 pp.

[6] A. Choudhry, Arithmetic progressions on Huff curves, J. Integer Seq. 18 (2015), Article 15.5.2., 9 pp.

[7] G. S. Çelik and G. Soydan, Elliptic curves containing sequences of consecutive cubes, Rocky Mountain J. Math. 48 (2018), 2163-2174.

[8] H. Edwards, A normal form for elliptic curves, Bull. Amer. Math. Soc. (N.S.) 44 (2007), 393-422.

[9] M. Kamel and M. Sadek, On sequences of consecutive squares on elliptic curves, Glas. Mat. Ser. III 52(72) (2017), 45-52.

[10] J.-B. Lee and W. Y. Vélez, Integral solutions in arithmetic progression for $y^{2}=x^{3}+k$, Period. Math. Hungar. 25 (1992), 31-49.

[11] A. J. Macleod, 14-term arithmetic progressions on quartic elliptic curves, J. Integer Seq. 9 (2006), Article 06.1.2., 4 pp.

[12] D. Moody, Arithmetic progressions on Huff curves, Ann. Math. Inform. 38 (2011), $111-116$.

[13] A. A. Ciss and D. Moody, Geometric progressions on elliptic curves, Glas. Mat. Ser. III $\mathbf{5 2 ( 7 2 )}$ (2017), 1-10.

[14] D. Moody, Arithmetic progressions on Edwards curves, J. Integer Seq. 14 (2011), Article 11.1.7., 4 pp.

[15] J. H. Silverman, The arithmetic of elliptic curves, Springer, Dordrecht, 2009.

[16] M. Stoll and J. E. Cremona, Minimal models for 2-coverings of elliptic curves, LMS J. Comput. Math. 5 (2002), 220-243.

[17] M. Ulas, A note on arithmetic progressions on quartic elliptic curves, J. Integer Seq. 8 (2005), Article 05.3.1., 5 pp.

[18] M. Ulas, On arithmetic progressions on genus two curves, Rocky Mountain J. Math. 39 (2009), 971-980.

\section{G. S. Çelik}

Department of Mathematics

Bursa Uludağ University

16059 Bursa

Turkey

E-mail: gamzesavascelik@gmail.com

M. Sadek

Faculty of Engineering and Natural Sciences

Sabancı University

34956 Tuzla, İstanbul

Turkey

E-mail: mmsadek@sabanciuniv.edu

G. Soydan

Department of Mathematics

Bursa Uludağ University

16059 Bursa

Turkey

E-mail: gsoydan@uludag.edu.tr

Received: 22.9.2018.

Revised: 5.3.2019. 